\title{
SOBRE A ESCOLHA DE PERMUTAÇÕES PARA FINS CRIPTOGRÁFICOS
}

\author{
R. M. Campello de Souza, A. N. Kauffman, R. C. C. Lima \\ Grupo de Pesquisas em Comunicações - CODEC \\ Departamento de Eletrônica e Sistemas - UFPE \\ C.P. 7800, 50970-730, Recife PE \\ ricardo@npd.ufpe.br
}

Resumo - Permutaçōes desempenhama um papel relevante no contexto de Criptografia, uma vez que representam uma importante contribuição no sentido de incrementar a difusāo gerada pelo processo de cifragem e muitos cripto-sistemas fazem uso das mesmas. Entretanto, poucas investigaçōes foram feitas no sentido de se elucidar aspectos de uma permutação que a tornem atrativa para fins criptográficos. Neste trabalho esta temática $e ́$ abordada e o problema da contagem do número đe permutaçōes sob $n$ elementos que fixam $i$ posições $\varepsilon$ considerado.

Abstract - Permutations play an important role in the field of data security, representing a substantial contribution to increase the diffusion of the encryption process and many cryptosystems make use of them . However, in general, the literature on the subject reports very few results concerning those aspects of a permutation that make it atractive for cryptographic purposes. In this paper the subject is considered and, in the search for cryptographically strong permutations to be used in private or public cryptosystems, the problem of counting the number of perrmutations of degree $n$ that fix $i$ elements is approached.

Palavras Chaves : criptografia, permutaçōes, chave privada.

\section{INTRODUÇÃO}

Permutações são elementos de atuação vigorosa, não apenas no contexto da Matemática, mas também em muitas aplicaçōes na Engenharia. Na área de segurança de dados, especificamente, as mesmas tem um papel importante na concepção de algoritmos de cifragem. De fato, os cripto-sistemas de chave privada mais seguros já concebidos empregama cifras do tipo produto, as quais implementam uma combinação de transformaçōes envolvendo substituiçōes e transposiçōes dos caracteres do bloco de informação a ser protegido. A transposição de um bloco de $\mathrm{n}$ caracteres $e$ efetuada através de uma permutação, a qual contribui para incrementar a difusão resultante da utilização de uma cifra. No projeto de cifras de bloco, os princípios de confusão e difusāo, enunciados por Shannon em 1949 [1], permanecem ainda como a principal orientação a ser seguida.

As cifras de bloco mais comumente expregadas nas modernas redes de computadores de alta velocidade fazem uso de permutações no algoritmo de cifragem. Um exemplo clássico e o bem conhecido padrão de cifragem de dados DES 28

\begin{tabular}{|c|c|c|}
\hline Cripto-sistema & Tipo de chave & $\#$ \\
\hline DES & Privada & 3 \\
\hline Gost & Privada & 8 \\
\hline McEliece & Pública & 1 \\
\hline Surto & Privada & 1 \\
\hline SAFER+ & Privada & 1 \\
\hline
\end{tabular}

TAB. 1: Alguns cripto-sistemas que empregam permutaçōes.

(Data Encryption Standard), o qual faz uso de três permutaçôes de graus diferentes [2]. Um exemplo mais recente $\varepsilon$ o cripto-sistema SAFER+ (Secure and Fast Encryption Routine), candidato ao padrão AES (Advanced Encryption Standard) que substituirá o DES [3]. SAFER+é uma cifra iterativa que emprega uma permutação sobre blocos de 16 caracteres construída de modo a produzir o máximo de difusāo no mínimo de rodadas [4].

Cifras baseadas em códigos para controle de erros, quer sejam de chave pública [5] ou privada [6], se fundamentam na modificação da estrutura de um cơdigo linear, modificação esta que usualmente preserva apenas sua linearidade. Para se obter este efeito de perda de estrutura, uma permutação $e$ aplicada durante o processo de cifragem [7]. A tabela 1 lista alguns cripto-sistemas que empregam pexmutaçōes (\# denota o número de permutaçōes em cada cripto-sistema).

A busca por permutaçōes fortes do ponto de vista de criptografia, isto $\varepsilon$, perrnutações que permitam estabelecer níveis específicos desejáveis de segurança computacional, tem sido pouco explorada na literatura [8], [9]. Neste trabalho o tema $e$ abordado e alguns resultados são obtidos acerca do comportamento de permutações de grau ne de sua utilização para fins criptográficos. Na próxima seção alguns fatos básicos sobre permutaçōes são apresentados e uma nova função aritmética, denotada $P_{n}(i)$, é introduzida e algumas de suas propriedades são estabelecidas. A seção 3 contém o principal resultado da pesquisa relatada neste trabalho, a completa especificação da função $P_{n}(i)$. Na seção 4 uma análise de comaportamento assimptótico é apresentada. A seção 5 apresenta alguns exemplos e as conclusões do trabalho são apresentadas na seçāo 6. 


\section{PRELIMINARES}

Definição 1 - Üma permutação de um conjunto $S$ é uma bijeçäo de $S$ em $S$. O grau de uma permutação é a cardinalidade do conjunto sobre o qual a mesma esta definida.

Sem perda de generalidade, no que se segue, considera-se $S$ como sendo o conjunto de inteiros $(1,2,3, \cdots, n)$. Dessa forma, se $p$ é uma permutação definida em $S$, a imagem do inteiro $i \in S$ É denotada por $p(i)$. Pode-se então usar a seguinte lista de pares $(i, p(i))$ para especificar $p$ :

$$
\left(\begin{array}{cccc}
1 & 2 & 3 & \cdots n \\
p(1) & p(2) & p(3) & \cdots p(n)
\end{array}\right)
$$

Uma notação mais compacta é obtida através do uso de ciclos. Se $a_{1}, a_{2}, a_{3}, \cdots, a_{k} \in S$, então $\left(a_{1} a_{2} a_{3} \cdots a_{k}\right)$ denota a permutação dos elementos de $S$ onde

$$
a_{1} \rightarrow a_{2}, \quad a_{2} \rightarrow a_{3}, \cdots, a_{k-1} \rightarrow a_{k}, \quad a_{k} \rightarrow a_{1}
$$

e $i \rightarrow i$, para todos os outros valores de $i \in S$. Esta permutação é chamada um ciclo (ou $k$-ciclo) e implica que cada elemento de $S$ aparece apenas uma vez em um único ciclo. Um ciclo com $k$ elementos é dito ter comprimento $k$.

Definição 2 - O conjunto das $n$ ! permutaçōes de $S$ juntamente com a operação de composição de permutações (funfões) é um grupo de permutaçōes denominado o grupo simétrico de grau $n$ e denotado por $S_{n}$.

Mostra-se que, em $S_{n}$, a ordem de uma permutação, isto $\ell$, o menor inteiro $r$ tal que $p^{r}=e$ (o mapeamento identidade), é dado pelo mínimo múltiplo comum dos comprimentos de seus ciclos [10]. Dessa forma, em $S_{3}$, o grupo simétrico de grau 3, os elementos tem ordem 1 , 2 e 3 . As $3 !=6$ permutaçōes de $S_{3}$ são $p_{1}=e, p_{2}=(12), p_{3}=(13), p_{4}=(23)$, $p_{5}=(123)$ e $p_{6}=(132)$.

A familia de funçōes aritméticas $P_{n}(i)$ definida a seguir, desempenha um papel relevante na escolha de permutações para fins criptográficos.

Definição $3-P_{n}(i)$ denota o número de permutaçōes de grau $n$ que fixam $i$ elementos, $0 \leq i \leq n, n \geq 1$.

Algumas propriedades simples da funçāo $P_{n}(i)$ são listadas a seguir.

Propriedade 1) $P_{n}(n)=1$ (Apenas a permutaçāo identidade fixa todos os elementos).

Propriedade 2) $P_{n}(n-1)=0$ (Nenhuma permutaçāo muda apenas um único elemento. De fato, excetuando-se a permutação identidade, qualquer permutação muda pelo menos duas posiçōes).

Propriedade 3) $\sum_{i=0}^{n} P_{n}(i)=n$ !

Um papel de destaque é reservado à $P_{n}(0)$, conforme mostra o lema 1

Lema $1-P_{n}(i)=\left(\begin{array}{c}n \\ i\end{array}\right) P_{n-1}(0), \quad 0 \leq i \leq n-1$
Prova :Existem $\left(\begin{array}{c}n \\ i\end{array}\right)$ maneiras de se escolher $i$ elementos

dentre os $n$. Para cada uma delas, os restàntes $(n-1)$ elementos mudam todos de posição, o que pode ser feito, por definiçāo, de $P_{n-1}(0)$ maneiras.

O lema 1, apesar de simples, desempenha um papel importante no sentido de caracterizar inteiramente a funçāo $P_{n}(i)$. Para atingirmos esse objetivo, é necessário determinar $P_{n-1}(0)$ ou, em geral, $P_{n}(0)$. Nesse ponto é importante observarmos que, do ponto de vista de criptografia, claramente os mais relevantes $P_{n}(i)$ 's săo aqueles para os menores valores de $i . O$ lema 2 a seguir mostra que $P_{n}(0)$ pode ser obtida como solução de uma equação de diferenças de segunda ordem com coeficientes variáveis.

Lema $2-P_{n}(0)$ satisfaz à equação de diferenças

$$
P_{n}(0)=(n-1)\left[P_{n-1}(0)-P_{n-2}(0)\right]
$$

com condiçōes iniciais e $P_{1}(0)=0$ e $P_{2}(0)=1$.

Prova : Uma verificação direta mostra que as conđiçōes iniciais são satisfeitas. Sem perda de generalidade, representamos os elementos a serem permutados pelas coordenadas $1,2, \ldots, n$. Focalizando nossa atenção em dois tais elementos, digamos 1 e $j, j 2,3, \ldots ., n$, existem duas situaçōes a serem levadas em conta :

i) 1 e $j$ trocam de lugares e então permanecem fixos. Desde que existem $(n-1)$ escolhas para $j$ e $(n-2)$ elementos permanecern para serem permutados, a contribuição é portanto $(n-1) P_{n-2}(0)$.

ii) Agora 1 mapeia em $j$, mas nāo vice-versa. Então tudo se passa como se estivéssemos partindo de (i) após a transposição de 1 e $j$. Novamente existem $(n-1)$ pontos de partida para $j$, mas agora $(n-1)$ elementos restam para serem (todos) permutados. Isto contribui com $(n-1) P_{n-1}(0)$. Adicionando-se as contribuições em $(i)$ e (ii), a relação desejada é obtida.

\section{SOLUÇÃO DA EQUAÇÃO DE DI- FERENÇAS}

De acordo com o lema 1, para explicitarmos a função $P_{n}(i)$ precisamos encontrar primeiramente $P_{n}(0)$. Isso é feito no lema 3 a seguir.

Lema $3-P_{n}(0)=n ! \sum_{j=0}^{n} \frac{(-1)^{j}}{j !}$

Prova : Por indução :

i) Para $n=1$ e $n=2$ obtemos, respectivamente, $P_{1}(0)=$ 0 e $P_{2}(0)=1 \mathrm{e}$, que atende às condiçōes iniciais do lema 2.

ii) Passo de indução: Do lema 2, considerando válida a proposição para $n$ e $n-1$, podemos escrever

$P_{n-1}(0)=n\left[n ! \sum_{j=0}^{n} \frac{(-1)^{j}}{j !}+(n-1) ! \sum_{j=0}^{n-1} \frac{(-1)^{j}}{j !}\right]$ 
Expandindo a expressāo e adicionando o termo correspondente a $j=n$ no segundo somatório, chegamos a

$$
P_{n-1}(0)=n !\left[n \sum_{j=0}^{n} \frac{(-1)^{j}}{j !}+\sum_{j=0}^{n} \frac{(-1)^{j}}{j !}-\frac{(-1)^{n}}{n !}\right]
$$

ou seja

$$
P_{n-1}(0)=(n+1) ! \sum_{j=0}^{n} \frac{(-1)^{j}}{j !}+(n+1) ! \frac{(-1)^{n+1}}{(n+1) !}
$$

e entāo

$$
P_{n+1}(0)=(n+1) ! \sum_{j=0}^{n+1} \frac{(-1)^{j}}{j !}
$$

de modo que o resultado é válido para $n+1$, o que conclui o passo de indução e completa a prova.

De posse dos lemas 1,2 e 3 determinamos $P_{n}$ (i) Trata-se de um resultado novo que tem implicaçōes interessantes.

Teorema $1-P_{n}(i)=\frac{n !}{i !} \sum_{j=0}^{n-i} \frac{(-1)^{j}}{j !}, \quad i=0,1, \ldots, n$.

Prova : Dos lemas 1 e 3 , obtemos

$$
P_{n}(i)=\left(\begin{array}{c}
n \\
i
\end{array}\right)(n-i) ! \sum_{j=0}^{n-i} \frac{(-1)^{j}}{j !}
$$

e o resultado segue-se após a expansāo do coeficiente binomial

Esse resultado indica que, para um dado valor fixo de $n$, os maiores valores de $P_{n}(i)$ correspondern a $i=0$ ou $i=1$. De fato, como mostra o corolário $1, P_{n}(i)$ é máximo para $i=0$ ou $i=1$, conforme $n$ seja, respectivamente, par ou ímpar.

Colorário $1-P_{n}(0)-P_{n}(1)=(-1)^{n}$.

Prova : Do teorema 1, podemos escrever

$$
P_{n}(0)=n ! \sum_{j=0}^{n} \frac{(-1)^{j}}{j !}
$$

e

$$
P_{n}(1)=n ! \sum_{j=0}^{n-1} \frac{(-1)^{j}}{j !}
$$

de modo que

$$
\begin{aligned}
P_{n}(0)-P_{n}(1) & =n !\left(\sum_{j=0}^{n} \frac{(-1)^{j}}{j !}-\sum_{j=0}^{n-1} \frac{(-1)^{j}}{j !}\right)= \\
& =\left.n !\left(\frac{(-1)^{j}}{j !}\right)\right|_{j=n}
\end{aligned}
$$

e o resultado segue.

A Tabela 2 mostra alguns valores de $P_{n}(i)$. De especial interesse para criptografia é o comportamento de $P_{n}(i)$ para. $i=0$ Por razōes de segurança é claramente desejável que seja usado, no processo de cifragem, uma das $P_{n}(0)$ permutaçōes que năo fixa nenhum elemento. Entretanto, isto pode ser feito de maneira prática e segura apenas se nós tivermos uma clara compreensão do comportamento relativo de $P_{n}(i)$ e $n !$.

\begin{tabular}{|c|c|c|c|c|c|c|c|c|c|}
\hline & $\mathrm{n}=1$ & 2 & 3 & 4 & 5 & 6 & 7 & 8 & 9 \\
\hline $\mathrm{i}=0$ & 0 & 1 & 2 & 9 & 44 & 265 & 1854 & 14833 & 133496 \\
\hline 1 & - & 0 & 2 & 8 & 45 & 264 & 1855 & 14832 & 133497 \\
\hline 2 & - & 1 & 0 & 6 & 20 & 135 & 924 & 7420 & 66744 \\
\hline 3 & - & - & 1 & 0 & 10 & 40 & 315 & 2464 & 22260 \\
\hline 4 & - & - & - & 1 & 0 & 15 & 70 & 630 & 5544 \\
\hline 5 & - & - & - & - & 1 & 0 & 21 & 112 & 1134 \\
\hline 6 & - & - & - & - & - & 1 & 0 & 28 & 42 \\
\hline 7 & - & - & - & - & - & - & 1 & 0 & 36 \\
\hline 8 & - & - & - & - & - & - & - & 1 & 0 \\
\hline 9 & - & - & - & - & - & - & - & - & 1 \\
\hline
\end{tabular}

TAB. 2: Alguns valores de $P_{n}(i)$.

\section{COMPORTAMENTO ASSIMPTÓ- TICO}

O corolário 2 a seguir mostra um resultado interessante sobre o comportamento relativo assimptótico das funçōes $P_{n}(i)$ e $n !$.

Colorário 2 $\lim _{n \rightarrow \infty}\left(\frac{n !}{P_{n}(i)}\right)=i$ !e.

Prova : A expansão em série de Maclaurin da funç̧ão exponencial $f(x)=e^{-x}$ é

$$
e^{-x}=\sum_{j=0}^{\infty} \frac{(-1)^{j} x^{j}}{j !}
$$

Do teorema 1

$$
\frac{P_{n}(i)}{n !}=\frac{1}{i !} \sum_{j=0}^{n-i} \frac{(-1)^{j}}{j !}
$$

de modo que para $x=l$ e considerando o limite quando $n \rightarrow \infty$, obtemos

$$
\lim _{n \rightarrow \infty}\left(\frac{P_{n}(i)}{n !}\right)=\frac{e^{-1}}{i !}
$$

e o resultado segue.

Esse corolário mostra que o número e pode ser obtido através da função $P_{n}(0)$ (ou $P_{n}(1)$ ). Isto porque, fazendo-se $i=0$ (ou $i=1$ ) no limite acima, resulta $\mathrm{em}$

$$
\lim _{n \rightarrow \infty}\left(\frac{n !}{P_{n}(0)}\right)=e
$$

A Tabela 3 mostra alguns valores da relação $R=$ $n ! / P_{n}(0)$, de onde se pode perceber a rapidez do processo de convergência (na $10^{\circ}$ iteração, o valor obtido está correto a uma precisão de $10^{-6}$ ).

$\mathrm{O}$ corolário 2 fomece algumas indicações úteis sobre a viabilidade de se usar uma das $P_{n}(0)$ permutaçōes que não fixam nenhum elemento. Especificamente, ele mostra que, 


\begin{tabular}{|c|c|c|c|}
\hline 1 & $\infty$ & 6 & 2,7169811 \\
\hline 2 & 2,0000000 & 7 & 2,7184466 \\
\hline 3 & 3,0000000 & 8 & 2,7182633 \\
\hline 4 & 2,6666666 & 9 & 2,7182837 \\
\hline 5 & 2,7272727 & 10 & 2,7182817 \\
\hline
\end{tabular}

TAB. 3: Alguns valores de $R=n ! / P_{n}(0)$.

para um dado valor fixo de $n$ suficientemente grande (e.g., $n \geq 10$ ), o número de permutaçōes que fixam $i$ elementos, $i=2,3,4, \ldots$, decresce, de forma monotônica, com percentuais em relaçāo ao total de permutaçōes de, respectivamente, $18,39 \%, 6,13 \%, 1,53 \%$, etc. Dessa forma vemos que, mesmo para valores moderados de $n$ (Tabela 2), $P_{n}(0)$ e $P_{n}(1)$ representam, cada uma, aproximadamente $(100 / R) \%=36,7 \%$ de todas as $n$ ! permutaçōes de grau $n$. Considerando os recursos computacionais do criptoanalista bem como aspectos práticos de implementação, esta informação pode ser usada no projeto de cripto-sistemas que fazem uso de permutaçōes como parte de sua chave privada .

\section{EXEMPLOS}

Nesta seção alguns exemplos simples são apresentados visando ilustrar alguns dos resultados obtidos anteriormente.

Exemplo 1 - A difusão ótima em uma cifra iterativa é obtida quando qualquer símbolo de texto claro influencia todos os símbolos de texto cifrado em um número mínimo de rodadas. O embaralhamento armênio $(E a)$, responsável pela difusão ótima no cripto-sistema SAFER+ é um elemento de ordem 70 do grupo simétrico $S_{1} 6$. Especificamente

$$
E a=\left(\begin{array}{lllllll}
1 & 13 & 3 & 5 & 15 & 11 & 9
\end{array}\right)\left(\begin{array}{lllll}
2 & 6 & 8 & 14 & 12
\end{array}\right)\left(\begin{array}{ll}
4 & 16
\end{array}\right),
$$

onde os elementos 7 e 10 são mantidos fixos.

Exemplo 2 - A seguir estão listadas todas as $4 !=24$ permutaçōes de $S_{4}$, agrupadas de duas formas, a saber (1) em classes de elementos de mestma ordern $R_{4}(r), r=1,2,3,4$ e (2) em classes $F_{4}(i)$ correspondentes aos valores de $P_{4}(i)$ para $i=0,1,2,4\left(F_{4}(3)=0\right)$ :

$$
\begin{aligned}
& R_{4}(1)=\{(1)(2)(3)(4)=e\} . \\
& R_{4}(2)=\{(12)(34),(13)(24),(14)(23),(12),(13),(14) \text {, } \\
& \text { (23), (24), (34)\}. } \\
& R_{4}(3)=\{(123),(132),(124),(142),(134),(143),(234), \\
& (243)\} \text {. } \\
& R_{A}(4)=\{(1234),(1243),(1324),(1342),(1423), \\
& \text { (1432)\}. } \\
& F_{4}(0)=\{(12)(34),(13)(24),(14)(23),(1234),(1243), \\
& (1324),(1342),(1423),(1432)\} \text {. } \\
& F_{4}(1)=\{(123),(132),(124),(142),(134),(143),(234), \\
& (243)\} \text {. } \\
& F_{4}(2)=\{(12),(13),(14),(23),(24),(34)\} \text {. } \\
& F_{4}(4)=\{(1)(2)(3)(4)=e\} \text {. }
\end{aligned}
$$

Observa-se que as 6 permutaçōes de maior ordem em $S_{4}$ pertencem todas à classe $F_{4}(0)$, isto é, são permutações que não fixam nenhum elemento.

\section{CONCLUSÕES}

Permutaçōes tem sido empregadas em cripto-sistemas simétricos ou assimétricos, fazendo parte, ou não, da chave do sistema. Em qualquer caso, o objetivo principal é propiciar a difusão dos símbolos de texto claro sobre o texto cifrado, em consonância com o princípio da difusão de Shannon. Nesse contexto, este trabalho analisa aspectos relativos à escolha de permutações de grau $n$, visando sua utilização em criptografia. Inicialmente, uma nova famúlia de funçōes aritméticas $P_{n}(i)$, indexada em $i, i=0,1, \ldots, n$, foi introduzida, a qual indica o número de permutaçōes de grau $n$ que fixam $i$ elementos. Visando o objetivo de utilizar aquelas permutações que mais contribuam para aumentar a difusão do processo de cifragem, tomou-se necessário explicitar analiticamente $P_{n}(i)$ e avaliar seu comportamento para valores específicos de $i$. Algumas de suas propriedades foram estabelecidas e foi mostrado que $P_{n}(i)$ satisfaz uma equação de diferenças com coeficientes variáveis, cuja solução foi então obtida. Dessa forma, foi possível estabelecer uma avaliação quantitativa que tem implicaçōes para o projeto de cripto-sistemas que empregam permutaçōes em sua chave privada. O comportamento assimptótico de $P_{n}(i)$ foi analisado e mostrou-se que a relação entre o número total de permutaçōes de grau $n$ e $P_{n}(0)$ converge para o numero $e$, a base do sistema de logaritmos neperianos.

\section{AGRADECIMENTOS}

Os autores agradecem as valiosas sugestōes dadas pelo Professor Hélio M. de Oliveira durante a preparação deste trabalho.

\section{REFERÊNCIAS}

[1] C. E. Shannon, "Communication Theory of Secrecy Systems", Bell System Tech. J., vol. 28, pp. 656-715, Oct., 1949.

[2] National Bureau of Standards, NBS FIPS PUB 46, "Data Encryption Standard", US Department of Commerce, January 1977.

[3] Advanced Encryption Standard (AES) - A Crypto Algorithm for the Twentyfirst Century, http ://www.nist.gov/aes, http ://www.cylink.com/SAFER.

[4] J. L. Massey, "On the Optimality of SAFER+ Diffusion", Second Advanced Encryption Standard Candidate Conference, Rome, Italy, March 22-23, 1999.

[5] R. J. McEliece, "A Public-Key Cryptosystem Based on Algebraic Coding Theory", DSN Progress Report 4244, pp. 114-116, Jet Propulsion Laboratory, Pasadena, CA, Jan./Feb. 1978.

[6] R.M. Campello de Souza, J. Campello de Souza, "Array Codes for Private-Key Encryption", Electronics Letters, vol. 30, No. 17, pp. 1394 - 1396, August 1994. 
[7] R. M. Campello de Souza, J. Campello de Souza, "C6digos Produto Multiníveis Para Correção de Erros BiSeparáveis com Aplicações em Criptografia", Revista Brasileira de Telecomunicações, vol. 10, No. 1, pp. 7 - 14, dezembro 1995.

[8] P. Mathys, "On the Specification of Permutations for Block Ciphers", Proceedings of the IEEE International Symposium on Information Theory, pg. 232, January 1990.

[9] L. Mittenthal, "A Source of Cryptographically Strong Permutations for Use in Block Ciphers", Proceedings of the IEEE International Symposium on Information Theory, pg. 233, January 1990.

[10] J. R. Durbin, "Modem Algebra", 3rd. Ed., JohnWiley, 1992.

Ricardo Menezes Campello de Souza formou-se em Engenharia Elétrica pela Universidade Federal de Pernambuco em 1974, obteve o título de Mestre em Ciências pela mesma Universidade em 1979 e o titulo de $\mathrm{PhD}$ pela University of Manchester, Inglaterra, em 1983, ambos em Engenharia Elétrica. Desde 1979 é Professor do Departamento de Eletrônica e Sistemas da UFPE, onde foi coordenador do Programa de Pós-graduaçāo em Engenharia Elétrica no período 19841987, Chefe do Departamento no periodo 1987-1992 e atualmente ocupa a posiçāo de Professor Adjunto. Seus interesses de pesquisa incluem matemática discreta, criptografia, teoria algebrica da cođificação e processamento digital de sinais.

André Neumann Kauffman formou-se em Engenharia Elétrica, modalidade Eletrônica (cum laude), pela Universidade Federal de Pernambuco em 1997, onde cursa atualmente o programa de Mestrado em Engenharia Elétrica. Seus interesses de pesquisa incluem matemática discreta, criptografia e processamento digital de sinais.

Rossana Claudia Cursino Lima é concluinte do Curso de Graduação em Engenharia Elêtrica, modalidade Eletrônica, da Universidade Federal de Pernambuco. Seus interesses de pesquisa incluem criptografia, processamento digital de sinais e comunicaçōes moveis. 